\title{
ON REGULAR FUNCTIONS FOR CERTAIN MARKOV PROCESSES
}

\author{
RICHARD ISAAC ${ }^{1}$
}

We consider temporally discrete Markov processes $\left\{X_{n}, n \geqq 0\right\}$ with arbitrary state space $\Omega$ and $\sigma$-field of events $\Sigma$. The processes to be studied possess a $\sigma$-finite stationary measure, i.e., a measure $Q$ such that $\int P(x, E) Q(d x)=Q(E)$ for all $E \in \Sigma$. $P(x, E)$ and $P^{k}(x, E)$ are the first and $k$ th step transition probabilities respectively [3, p. $190 \mathrm{ff}$.]. Throughout this note unless the contrary is explicitly asserted, statements holding almost surely (a.s.) refer to $Q$ measure. The transformation $P f=h$ is given by $\int P(x, d y) f(y)=h(x)$ when this operation is defined. Call a function $f$ regular or excessive if $P f=f$ or $P f \leqq f$ respectively, these relations holding a.s.

Given a class $D$ of functions, one would like to describe the functions in $D$ which are regular. Let $L_{1}$ and $L_{\infty}$ be the classes of integrable and a.s. bounded $\Sigma$-measurable functions on $\Omega$, and $J^{+}$the class of a.s. extended real valued nonnegative functions (these classes taken with respect to $Q$-measure). We say that condition (B) holds if $P\left(X_{n} \in E\right.$ i.o. $\left.\mid X_{0}=x\right)=1$ a.s. $(Q)$ on $\Omega$ whenever $Q(E)>0$ [4]. Our main result is

THEOREM 1. Let $D=L_{1} \cup L_{\infty} \cup J^{+}$. If condition (B) holds then the only regular functions in $D$ are the constant functions a.s.

Proof. Represent the process, as usual, on the set $\Omega_{0}$ of unilateral sequences $\omega=\left(\omega_{0}, \omega_{1}, \cdots\right)$ on which we consider the usual $\sigma$-field $\Sigma_{0}$ over the cylinder sets and obtain a measure $Q_{0}$ induced on $\Sigma_{0}$ using $Q$ and the transition probabilities [4]. If $T$ is the shift: $(T \omega)_{2}=\omega_{i+1}$, then $Q_{0}\left(T^{-1} S\right)=Q_{0}(S)$ for all $S$. $Q_{0}$ will be $\sigma$-finite since $Q$ is. Observe that we can always construct an equivalent probability measure $\bar{Q}_{0}$ on $\Sigma_{0}$ with $\bar{Q}_{i 1}(S) \leqq Q_{0}(S)$ provided $Q_{0}\left(\Omega_{i j}\right)=\infty$. For, let $\left\{E_{i}\right\}$ be a disjoint $\sigma$-finite decomposition of $\Omega$ for $Q$, and let $\left\{a_{i}\right\}$ be a sequence of positive numbers, each less than 1 , so that $\sum a_{i} Q\left(E_{i}\right)=1$. Then $\bar{Q}(E)=\sum a_{i} Q\left(E \cap E_{i}\right)$ defines a probability on $\Sigma$ inducing $\bar{Q}_{0}$ on $\Sigma_{0}$ and $\bar{Q}_{0}$ vanishes on the same sets as $Q_{0}$. It will be convenient to have $\bar{Q}_{0}$ at our disposal since the classical martingale theorems are

Received by the editors February 3, 1966.

${ }^{1}$ Research sponsored by the Air Force Office of Scientific Research, Office of Aerospace Research, U. S. Air Force, under AFOSR Grant No. 396-63 to Cornell University. 
phrased in terms of probability spaces. We will then be able to use these theorems directly rather than having to state relevant generalizations for infinite measure spaces.

Case 1: $f \in L_{\infty}$. This part of the proof does not require the existence of a stationary $Q$ nor condition (B). All that is needed is the hypothesis of ergodicity, i.e., if $\mu$ is the basic probability measure, then $T^{-1} S=S$ implies $S$ is trivial (up to $\mu$ equivalences). The proof follows Blackwell [1]. $f$ regular implies $E\left(f\left(X_{n}\right) \mid X_{n-1}\right)=f\left(X_{n-1}\right)$ so $f\left(X_{n}\right)$ is a bounded martingale converging to a limiting bounded $g$. Since $f\left(X_{n}(T \omega)\right)=f\left(X_{n+1}(\omega)\right), g$ is invariant: $g(T \omega)=g(\omega)$ a.s. $(\mu)$, and by ergodicity reduces to a constant a.s. $(\mu)$. By the uniform boundedness, the martingale theorem yields $E\left(g \mid X_{0}\right)=f\left(X_{0}\right)$ so that $f$ is constant a.s. $(\mu)$.

Case 2: $f \in J^{+}$. Suppose, first, that $f$ is a.s. finite valued. $f\left(X_{n}\right)$ is no longer necessarily a martingale since $f$ may not be integrable. exp $\left(-f\left(X_{n}\right)\right)$ is a bounded nonnegative submartingale, however, converging to a bounded invariant limit $g$ which reduces to a constant $d$ because condition (B) implies ergodicity, that is, the shift transformation $T$ on sequence space $\left(\omega_{0}, \omega_{1}, \cdots\right)$ has only $Q$ trivial invariant sets.

The submartingale inequality easily shows $d \neq 0$, and going to the limit under the integral sign yields $\lim _{n \rightarrow \infty} f\left(X_{n}\right)=c=-\log d$. To prove $f(x) \equiv c$ a.s., let $K=[x:|f(x)-c|>\epsilon]$ have positive $Q$-measure for some $\epsilon>0$. Since $f\left(X_{n}\right) \rightarrow c$, there is a set $E$ of sequences with $\left|f\left(X_{n}(\omega)\right)-c\right|<\epsilon / 2$ for $n>N$ and $Q_{0}(E)>0$. This contradicts condition (B) which implies that a.s. $\left(Q_{0}\right)$ on $E,\left|f\left(X_{n}\right)-c\right|>\epsilon$ for infinitely many $n$. Thus $|f(x)-c|<\epsilon$ for all $\epsilon>0$ a.s. and $f(x) \equiv c$ a.s. For general $f$ define $u=\min [f, k]$ for each constant $k \geqq 0$. $u$ is finite valued and in $J^{+}$and regular (see Theorem 3 ). The result readily follows.

Case 3: $f \in L_{1}$. We check $\sup _{n} E\left|f\left(X_{n}\right)\right|<\infty$ where the expectation can be considered with respect to the measure $\bar{Q}_{0}$ (if $Q_{0}$ is finite put $\left.\bar{Q}_{0}=Q_{0}\right)$. We have

$$
\int\left|f\left(X_{n}(\omega)\right)\right| \bar{Q}_{0}(d \omega) \leqq \int\left|f\left(X_{n}(\omega)\right)\right| Q_{0}(d \omega)=\int|f(x)| Q(d x)<\infty
$$

so that the martingale $f\left(X_{n}\right)$ converges to an invariant limit, i.e., a constant a.s., and the argument is concluded as in Case 2.

REMARKs. Blackwell has considered ergodic Markov processes with discrete state space and obtained Case 1 [1]. Spitzer discusses Cases 1 and 2 for aperiodic random walk [7, p. 276] and for aperiodic, recurrent random walk $[7$, p. 133] respectively (aperiodicity implies ergodicity). Theorem 1 includes all of these results since the recurrent 
random walks always have stationary measures $Q$ satisfying condition (B). Spitzer's discussion of Case 1 is a specialization of a very general theorem of Choquet and Deny [5] applicable to random walks on groups. Case 3 does not seem to have been noticed before in any special situations. For an example of a nonconstant regular function for a process satisfying condition (B), consider simple random walk on the integers, i.e., probability of displacement a unit step to the right or the left is $\frac{1}{2} . Q(\{x\})=1$ at each integer point $x$ gives a stationary measure, and the random walk is recurrent. $f(x)=x$ is a nonconstant regular function but $f \notin D$ as defined in Theorem 1 .

\section{Extensions and complements.}

(1) Suppose that condition (B) is strengthened to condition (C) by requiring that the basic statement of condition (B) hold at all points of $\Omega$ rather than only a.s. Call $f$ regular everywhere if $(P f)(x)=f(x)$ for all $x \in \Omega$. We inquire whether a stronger form of Theorem 1 is now true. Namely, if condition (C) holds and $f$ is regular everywhere, does $f \in L_{1} \cup L_{x} \cup J^{+}$imply $f \equiv c$ for all $x \in \Omega$ ? The answer is no; for a simple counter example consider random walk on the nonnegative integers with an absorbing barrier at zero. The transition probabilities are

$$
P(x,\{x+1\})=P(x,\{x-1\})=\frac{1}{2}, \quad x \geqq 1 ; \quad P(0,\{0\})=1 .
$$

A stationary measure for the process is $Q(\{0\})=1, Q(\{x\})=0, x \neq 0$ and condition (C) is satisfied here. The function $f(x)=x$ is in $L_{1} \cap L_{\infty}$ $\cap J^{+}$relative to $Q$, and is regular everywhere. We can, however, state the following result.

THEOREM 2. If condition (C) holds and $f$ is regular everywhere and bounded everywhere, then $f(x) \equiv c$ for all $x \in \Omega$.

Proof. According to Theorem $1, f(x) \neq c$ on a $Q$-null set $N_{1}$. For $k \geqq 2$, let $N_{k}=\left[x: P\left(x, N_{k-1}\right)>0\right]$. Then $U N_{k}$ is $Q$-null and its complement $M$ is stochastically closed, i.e., $P(x, M)=1$ for all $x \in M$. Moreover, $f(x)=c$ for $x \in M$. By condition (C), $P\left(f\left(X_{n}\right) \rightarrow c \mid X_{0}=x\right)=1$ for all $x \in \Omega$. By iterating the operator $P$ we obtain $\left(P^{n} f\right)(x)=f(x)$ for all $x$, or $E\left(f\left(X_{n}\right) \mid X_{0}=x\right)=f(x)$. If $f$ is bounded, the dominated convergence theorem may be used to take the limit under the expectation to obtain $f(x)=c$.

One can clearly phrase more general theorems by imposing conditions permitting passage to the limit under the above expectation.

(2) What can be said about the regular functions if we consider the operator $P$ obtained from a substochastic kernel rather than a stochastic one? Thus $P(x, \Omega) \leqq 1$ for all $x$, and it is natural to require 
the process to have a subinvariant measure $Q$ rather than a stationary measure (i.e., $\int P(x, E) Q(d x) \leqq Q(E)$ ). This appears to be an open question. If the process has the subinvariant measure $Q$ it is apparent, however, that a function $f$ with $P f=f$ a.s. $(Q)$ cannot reduce to a constant $c$ a.s. unless $P$ is a.s. stochastic. To see this, first observe that from $f=c$ a.s. we obtain $P(x, N)=0$ a.s. where $N$ is the $Q$-null set on which $f \neq c$. Hence, for almost every $x$ one obtains

$$
\begin{aligned}
f(x) & =\int_{\Omega} P(x, d y) f(y)=\int_{\Omega-N} P(x, d y) f(y)=c P(x, \Omega-N) \\
& =c P(x, \Omega) \leqq c
\end{aligned}
$$

a contradiction to the assumption $f=c$ a.s. unless $P(x, \Omega)=1$ a.s.

We say condition (A) holds if $P\left(X_{n} \in E\right.$ i.o. $\left.\mid X_{0}=x\right)=1$ a.s. for $x \in E$.

THEOREM 3. If condition (A) holds and if $f$ is real valued and nonnegative, then $f$ excessive implies $f$ regular.

Proof. Assume $f$ nonnegative excessive and suppose the set $A=[x:(P f)(x)+\epsilon<f(x)]$ has positive $Q$-measure for some $\epsilon>0$. $\sum_{k=1}^{n} P^{k}(x, A) \rightarrow \infty$ a.s. on $A$ by condition (A) so that

$$
\begin{aligned}
f & \geqq f-P^{n+1} f=\sum_{k=0}^{n} P^{k}(f-P f)=\int \sum_{k=0}^{n} P^{k}(x, d y)(f-P f)(y) \\
& \geqq \int \sum_{A} \sum_{k=0}^{n} P^{k}(x, d y)(f-P f)(y) \geqq \epsilon \sum_{k=0}^{n+1} P^{k}(x, A) \rightarrow \infty
\end{aligned}
$$

a.s. on $A$, a contradiction since $f$ is real valued and $Q(A)>0$.

This theorem also follows immediately from the fact that condition (A) implies $P$ is a conservative operator, i.e., the dissipative part (see [2]) is $Q$-null. We omit the proof of this statement. Theorem 3 then results since a conservative operator has no nontrivial potential (e.g. $[6$, p. 184]).

Notice that Case 2 of Theorem 1 could have been derived directly from Case 1 and Theorem 3 by considering the bounded excessive function $u(x)=\min [f(x), k]$ for any constant $k \geqq 0$, where $f \in J^{+}$ (cf. [7, p. 339, problem 1]).

COROLlary. Let condition (B) hold. Then the functions

$$
\liminf _{n \rightarrow \infty} P^{n}(x, E)=f(x) \text { and } \limsup _{n \rightarrow \infty} P^{n}(x, E)=g(x)
$$

reduce to constants a.s. for any fixed set $E$. If $E$ and $F$ are fixed sets with 
$Q(F)>0$ and $x_{0}$ is a fixed point, then

$$
\liminf _{n \rightarrow \infty} \frac{\sum_{k=1}^{n} P^{k}(x, E)}{\sum_{k=1}^{n} P^{k}\left(x_{0}, F\right)}=h(x)
$$

reduces to a constant, possibly infinity, a.s. for almost all choices of $x_{0}$. If condition (C) holds (see discussion after Theorem 1) then the assertion about $h(x)$ is valid for all choices of $x_{0}$.

Proof. An application of Fatou's Lemma and its variant for uniformly bounded functions shows $P f \leqq f$ and $P g \geqq g$, so it easily follows from Theorem 3 that $f$ and $g$ are regular and so must be constant by Theorem 1. Since $\sum_{k=1}^{n} P^{k}(x, F) \rightarrow \infty$ a.s. by condition (B) (excluding the $Q$-null set of $x_{0}$ on which $\sum_{k=1}^{n} P^{k}\left(x_{0}, F\right)$ converges), it is easily seen that $h$ is excessive and the proof is concluded as above. Under condition (C), $\sum_{k=1}^{n} P^{k}\left(x_{0}, F\right) \rightarrow \infty$ for all $x_{0} \in \Omega$, so that for all choices of $x_{0} h$ is excessive.

Suppose we only assume that the process possesses a finite stationary $Q$. Then Blackwell's proof shows $f \in L_{\infty}$ and regular has the representation $f=E\left(g \mid X_{0}\right)$ where $g$ is an invariant function. By stationarity $E\left(g \mid X_{0}\right)=g[3$, p. 460$]$ so that $f\left(X_{0}\right)$ is invariant. A possible conjecture might be: if $Q$ is a $\sigma$-finite stationary measure and $f \in D$ and regular, then $f\left(X_{0}\right)$ is an invariant function. If this statement were true we would obtain the assertion of Theorem 1 as an immediate consequence for any ergodic process. However, this conjecture is false, as the following counterexample shows. The transition probabilities are

$$
\begin{aligned}
& P(x,\{x+1\})=P(x,\{x-1\})=\frac{1}{2}, \quad x \geqq 1, \\
& P(x,\{x-1\})=1, \quad x \leqq 0,
\end{aligned}
$$

where the state space is the set of all integers. A stationary $Q$ is given by: $Q(\{x\})=x$ for $x \geqq 1, Q(\{x\})=\frac{1}{2}$ for $x \leqq 0$. Define $f(x)=x$ for $x \geqq 0, f(x)=0$ for $x<0$. Then $f(x)$ is nonnegative and regular. But the process is ergodic by [1], so that if $f\left(X_{0}\right)$ were invariant, $f(x)=$ constant a.s. $(Q)$, a contradiction. However, if there is some recurrence in the process the conjecture is true. Stated precisely, we have

THEOREM 4. Let condition (A) hold and let $Q$ be $\sigma$-finite stationary. Let $f \in D$ and regular. Then $f\left(X_{0}\right)$ is an invariant function a.s. $\left(Q_{0}\right)$.

Sketch of PROOF. Under condition (A), Doob's discussion [3, p. $458 \mathrm{ff}$.] may be generalized to infinite stationary measures. (It should 
be observed that finite stationary measures always satisfy condition (A) - this is simply a probabilistic form of the well-known Poincare Recurrence Theorem.) For each of the (sub) martingales arising in the three cases of Theorem 1, we will obtain $f\left(X_{n}\right) \rightarrow g$ a.s. where $g$ is invariant. Then $g$ is a function of $X_{0}: g=h\left(X_{0}\right)$ (if condition (A) is not valid, this statement is not necessarily true), and $f\left(X_{n}\right)-h\left(X_{n}\right) \rightarrow 0$. If $Q$ were finite, the invariance of $Q$ would imply $Q\left(\left|f\left(X_{n}\right)-h\left(X_{n}\right)\right|>\epsilon\right)$ $=0$ for all $\epsilon>0$, so that $f=h$ a.s. However, under condition (A), by restricting ourselves to subsets of positive, finite measure, it may still be concluded, as in the finite case, that $Q\left(\left|f\left(X_{n}\right)-h\left(X_{n}\right)\right|>\epsilon\right)=0$. Thus $f=h$ a.s. and $f\left(X_{0}\right)$ is invariant.

CoRollary. Under the hypotheses of Theorem 4 , if $Q$ is ergodic then condition (B) holds and Theorem 1 is valid.

Proof. The set ( $X_{n} \in E$ i.o.) is invariant, so that

$$
P\left(X_{n} \in E \text { i.o. } \mid X_{0}=x\right)=0 \text { or } 1 \text { a.s. }
$$

If $Q(E)>0$, condition (A) excludes the possibility that this quantity equals zero. Hence Theorem 1 is valid by virtue of our proof, or else Theorem 1 follows as a corollary to Theorem 4 since the only invariant functions are constants.

We thank Dr. J. B. Robertson for several helpful criticisms and Professor J. L. Doob for comments and suggestions.

\section{REFERENCES}

1. D. Blackwell, On transient Markov processes with a countable numbers of states and stationary transition probabilities, Ann. Math. Statist. 26 (1955), 654-658.

2. R. V. Chacon, Identification of the limit of operator averages, J. Math. Mech. 11 (1962), 961-968.

3. J. L. Doob, Stochastic processes, Wiley, New York, 1953.

4. T. E. Harris and H. Robbins, Ergodic theory of Markov chains admitting an infinite invariant measure, Proc. Nat. Acad. Sci. U.S.A. 39 (1953), 860-864.

5. G. Choquet and J. Deny, Sur l'equation $\mu=\mu * \sigma$, C. R. Acad. Sci. Paris 250 (1960), 799-801.

6. J. Neveu, Bases mathematique du calcul des probabilities, Masson, Paris, 1964.

7. F. Spitzer, Principles of random walk, Van Nostrand, Princeton, N. J., 1964.

Cornell University and

Hunter College 